\title{
A Working Definition of Online Citizen Science
}

\author{
Cathal Doyle ${ }^{1}$, Markus Luczak-Roesch ${ }^{1}$, Yevgeniya Li $^{1}$, Dayle Anderson ${ }^{1}$, Brigitte \\ Glasson $^{1}$, Prem Khanal ${ }^{1}$, Matthew Boucher ${ }^{2}$, Carol Brieseman ${ }^{3}$, Dianne Christenson ${ }^{4}$, and \\ Melissa Coton ${ }^{5}$ \\ ${ }^{1}$ Victoria University of Wellington \\ ${ }^{2}$ South Wellington Intermediate School \\ ${ }^{3}$ Hampton Hill School Tawa \\ ${ }^{4}$ Koraunui School Stokes Valley \\ ${ }^{5}$ Boulcott School Lower Hutt
}

May 5, 2020

\begin{abstract}
Citizen science, and online citizen science, are part of a movement towards open and participatory science, where education is particularly interested due to its potential benefits such as educating learners about the scientific process, as well as the topics of their study. This research is part of a project investigating the role of online citizen science in primary school science education, and provides an understanding of both citizen science and online citizen science from the literature, and then derives working definitions, which will be the guide for our further investigations.
\end{abstract}

\section{Introduction}

There has been a movement in recent years towards open and participatory science, in an effort to make scientific research more accessible to all levels of society. Citizen science (CS), and online citizen science (OCS) aim to help with this movement (Bonney et al., 2009), where the latter has become more and more popular in recent years (Nov et al., 2011). One area of society that has become particularly interested in OCS is that of education (especially science education) (Harlen, 2017), where potential benefits are to use OCS to educate learners about the scientific process, and about the particular topics of real scientific projects through participation facilitated by digital technologies. However, research on how OCS relates to the formal setting of science education has received little attention so far. Furthermore, while much research spoke about OCS, no work so far seems to offer an unambiguous differentiation between CS and OCS. This article aims to close this gap, first providing an understanding of both CS and OCS from the literature, and then deriving working definitions from these understandings, which will be the guide for our investigation of online citizen science in the science education of primary school children.

The remainder of this article is structured as follows. We begin by providing the background of our research project, emphasising the link to education research and teaching practice. Afterwards we describe the methodology we followed for our initial literature review from which we derived the working definitions of CS and OCS, which will be presented afterwards. Finally we give a brief outline of how this informs our ongoing research on novel ways to purposefully embed OCS for Year 3-8 students in New Zealand primary classrooms that meet the aims and intentions of the Nature of Science strand of the New Zealand Curriculum. 


\section{Citizen Scientists in the Classroom: Investigating the Role of Online Citizen Science in Primary School Science Education}

This research is part of a larger research project that has been funded by the Teaching and Learning Research Initiative (TLRI). The TLRI is a fund initiated by the New Zealand government to "link education research and teaching practice"(sit). The "Citizen Scientists in the Classroom" project explores the impact on student learning and engagement with science, incorporating OCS projects in New Zealand primary school classrooms (Year 3-8). It involves a co-constructive partnership (see Fig. 1) between researchers at Victoria University of Wellington and primary school teachers who have been identified as advocates of science education in New Zealand, and is the first attempt to investigate the potential of OCS projects to contribute to the improvement of science education of primary-age children.

This project is itself organised as a participatory open science project, and aims for a continuous interaction with the scientific community, educators, students and their parents, as well as the general public. For this reason we make as many resources of our work as possible publicly available, even when these are in a preliminary or work in progress stage. This working definition is the initial artefact that we want to make available, and for which we seek feedback, comments and input from all the aforementioned groups. By doing so we hope to achieve high transparency in the scientific process of this project, to contribute a blueprint or reference case of a novel open approach to scientific inquiries in the areas of educational research, computer supported collaborative work (CSCW) and human-computer interaction (HCI), and ultimately improve the outcomes of our projects for the benefit of teachers and learners.

\section{Citizen Science and Online Citizen Science: A Literature Review}

\section{Methodology}

The cornerstone of a good literature review is to apply a conceptual framework that helps to focus it (Willison and Siponen, 2007). We adopted the approach proposed by Webster and Watson (2002). This initially consisted of specifying the domain of interest for the research, which in this case is citizen science, and online citizen science. To gather relevant literature for this domain, a search was conducted on "Google Scholar" for the key terms "citizen science" and "online citizen science". From the opening search, a number of articles were identified, and an initial concept matrix was built, where the predetermined concepts were "citizen science" and "online citizen science". In addition to this we built on our previous knowledge about relevant scientific papers in this area and added those to our literature corpus. Each article was then reviewed, and added to the concept matrix anytime it referred to one of these predetermined concepts, inserting the particular sentence that corresponded to the concept. After this, the citations of these articles were reviewed to identify other relevant articles, and these were also read and added to the concept matrix when relevant. After the tenth article was reviewed, a saturation point had been reached where no new information about the concepts was being discovered. The completed concept matrix is presented in Table 1 below (or can be viewed as a Google Sheet here), which was analysed for emerging patterns to help create an understanding of CS and OCS.

\section{What is Online Citizen Science anyway? Two working definitions}

Before a definition of OCS can be formed, it is first important to understand what CS is, as it's the foundation on which OCS is built. This understanding is created from the concept matrix in Table 1, where the emerging patterns of what the articles discuss are synthesised. The same approach is then adopted to provide an understanding of OCS, and a working definition is again formed from this understanding. 


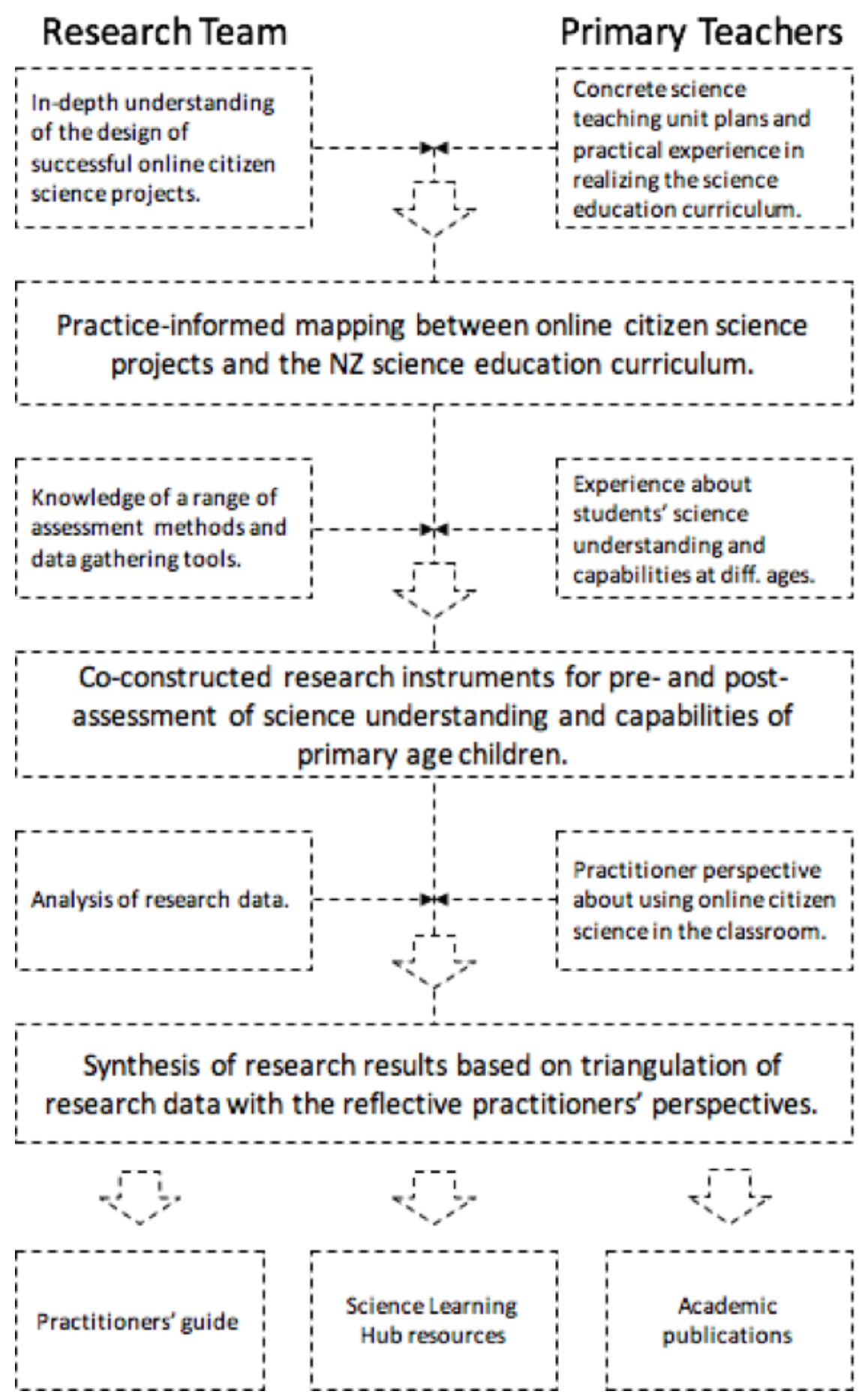

Figure 1: The research-practice nexus underlying our co-constructive partnership.

A working definition of Citizen science

Citizen science is a concept that has been practiced as far back as the 1700s (Raddick et al., 2009), and is a scientific practice (Raddick et al., 2009, 2013) that involves members of the public (Nov et al., 2011; 
Crowston et al., 2018; Hassman et al., 2013) actively engaging with professional scientists (Nov et al., 2011; Wiggins and Crowston, 2011; Raddick et al., 2013) in scientific work (Wiggins and Crowston, 2011). The members of the public participating can be referred to as citizen scientists (Raddick et al., 2009, 2013) or volunteers (Raddick et al., 2009; Wiggins and Crowston, 2011; Crowston et al., 2018). The engagement requires following an established protocol (Wiggins and Crowston, 2011) that is often created by the scientist (Lewenstein, 2004b) (although it can be argued that citizen scientists can participate in some of these steps), and can include one or more of the following tasks: data collection (Raddick et al., 2009; Wiggins and Crowston, 2011; Crowston et al., 2018); data processing (Curtis, 2014; Yadav and Darlington, 2017); data analysis \& interpretation (Raddick et al., 2009; Wiggins and Crowston, 2011; Crowston et al., 2018); and/or dissemination of results (Crowston et al., 2018; Lewenstein, 2004a). Outcomes from citizen science projects includes advancements in scientific research (Raddick et al., 2013; Hassman et al., 2013), as well as increasing the public's understanding of science (Raddick et al., 2009; Nov et al., 2011; Hassman et al., 2013). Based on this understanding of citizen science, we offer the following definition:

"Citizen science is a process that involves professional scientists and citizen scientists engaging on a scientific project. This engagement follows an established protocol, often created by the professional scientists, which can include one or more of the following tasks: data collection, data processing, data analysis \& interpretation, and/or dissemination of results. Outcomes should be advancements in scientific research, as well as an increase in the public's understanding of science."

While this provides us with an understanding, and definition of CS, online citizen science (OCS) differs in various ways. We suggest that it is important to highlight these differences, in particular in the light of increasingly digital learning environments. Thus, an overview of OCS is presented in the next section, along with a working definition.

\section{A working definition of Online citizen science}

Since the introduction of the Internet, and advancements in other digital technologies, citizen science has evolved and many CS projects are situated online (Raddick et al., 2009; Curtis et al., 2017), allowing professional scientists to be able to engage with citizen scientists in new ways (Raddick et al., 2009; Nov et al., 2011; Raddick et al., 2013). This includes being able to provide easier access to large datasets (Raddick et al., 2009; Hassman et al., 2013; Curtis et al., 2017); making tools available to support engagement from citizen scientists that are geographically distributed (Nov et al., 2011; Wiggins and Crowston, 2011; Hassman et al., 2013); facilitating communication in new ways between citizen scientists (Raddick et al., 2009); and providing a wider reach to a broader audience of citizen scientists (Nov et al., 2011; Wiggins and Crowston, 2011). These citizen science projects can be aided by technology (Wiggins and Crowston, 2011), or can be completely mediated online through technology (Wiggins and Crowston, 2011; Crowston et al., 2018). This extension of citizen science has been called online citizen science (Raddick et al., 2009; Crowston et al., 2018), and digital citizen science (Nov et al., 2011). Based on this understanding of online citizen science, we offer the following definition:

"Online citizen science is an extension of citizen science, where the tasks to be completed are aided, or completely mediated, through the Internet. Engagement can occur in different ways such as providing larger datasets to be analysed; making tools available to support engagement from citizen scientists that are geographically distributed; enabling new ways of communication between citizen scientists; and providing a wider reach to a broader audience of potential participants." 


\section{Conclusion}

In this article, which is a living open science resource, we presented an attempt to unambiguously define and differentiate citizen science and online citizen science. It is the outcome of the initial exchange between academic researchers at Victoria University of Wellington and partner teachers who are involved in science teaching in New Zealand primary schools. Because the difference between online and offline is a guiding rationale of our project, we decided to conduct a literature review, examining academic perspectives on the matter. By doing so we inform our next steps in the project and also seek to contribute to a better understanding in the research community and the general public of the difference between CS and OCS.

We suggest that it is important to make that difference, in particular in the light of increasingly digital learning environments and the role of digital technologies in the New Zealand Curriculum and similar primary and secondary school curricula. Emphasising that digital technologies are a utility for some scientific endeavours but not all of them (or not for all stages of them), bares a significant potential to create awareness of the fact that we always have to be critical when utilising almost ubiquitous technologies and ask whether the technologies are utilised purposefully. Furthermore, we seek to stimulate a discussion about the promises and perils of the digital trace that citizen scientists leave when engaging with CS projects online, which is an even bigger issue when the citizen scientists are students at schools. 


$\begin{array}{lcc}\begin{array}{cc}\text { Au- } \\ \text { thor }\end{array} & \text { Year } & \text { Title } \\ \text { Has- } & 2013 & \text { Learning at the } \\ \text { s- } & \text { Seafloor, Looking at } \\ \text { mann } & \text { the Sky: The } \\ \text { et } & \text { Relationship Between } \\ \text { al. } & \text { Individual Tasks and } \\ & \text { Collaborative } \\ & \text { Engagement in Two } \\ & \text { Citizen Science } \\ \text { Wig- } & \text { Projects } \\ \text { gins } & \text { From Conservation to } \\ \text { and } & \text { Crowdsourcing: A } \\ \text { Crow- } & \text { Typology of Citizen } \\ \text { ston } & \text { Science }\end{array}$

$\begin{array}{ll}\text { Crow-2018 } & \text { Coordinating } \\ \text { ston } & \text { Advanced Crowd } \\ \text { et } & \text { Work: Extending } \\ \text { al. } & \text { Citizen Science }\end{array}$

Rad- 2010 Galaxy Zoo: Exploring dick the Motivations of et Citizen Science

al. Volunteers

\section{Citizen Science}

The activities supported by citizen science projects have the potential to lead to significant scientific discoveries and to also support participant learning opportunities

Citizen science is a form of research collaboration involving members of the public in scientific research projects to address real-world problems

Active engagement in scientific work differentiates citizen science from other forms of public participation in scientific research where volunteers take less active roles

Citizen science is related to long-standing programs employing volunteer monitoring for natural resource management, and is often employed as a form of informal science education or outreach to promote public understanding of science

Citizen science is a broad term describing scientific projects that rely on contributions to scientific research from members of the public (i.e., citizens in the broadest sense of the word)

The practice of "citizen science" involves members of the public ("citizen scientists") working with professional scientists to complete a research project.

Citizen science pre-dates the Internet, and early projects involved participants making observations of the natural world that were often reported through the mail. Today, these
Online citizen science projects provide tools and opportunities that support public engagement in scientific research processes, often involving large data sets

Virtual modes of contribution make it possible for a broader audience to engage in scientific work, as in other open content projects. An increasing number and variety of citizen science projects are taking advantage of the affordances of technology to advance scientific research

Citizen science projects that are entirely mediated by

information and communication technologies (ICTs) are often considered a form of crowdsourcing applied to science

In the science-oriented Virtual projects, all project activities are ICT-mediated with no physical elements whatsoever, differentiating them from the Investigation projects in which the physical places of volunteer participation was also important

The interactions between volunteers and the project organizers are often via the Web, e.g. on a site that accepts contributed data (e.g., eBird) or that presents data and collects volunteers' annotations (e.g., Zooniverse)

The availability of large scientific datasets through the Internet has allowed citizen science projects to engage volunteers in new ways. In addition to collecting data, citizen scientists can analyze existing data, including data from modern astronomy surveys and missions

The Internet, however, allows citizen scientists to participate in research in completely new ways. For the first time, large numbers of people are being asked to analyze entirely online 


\section{References}

Teaching and Learning Research Initiative Web site. URL http://www.tlri.org.nz/.

Rick Bonney, Caren B. Cooper, Janis Dickinson, Steve Kelling, Tina Phillips, Kenneth V. Rosenberg, and Jennifer Shirk. Citizen Science: A Developing Tool for Expanding Science Knowledge and Scientific Literacy. BioScience, 59(11):977-984, dec 2009. doi: 10.1525/bio.2009.59.11.9. URL https://doi.org/ 10.1525\%2Fbio.2009.59.11.9.

Kevin Crowston, Erica Mitchell, and Carsten Østerlund. Coordinating Advanced Crowd Work: Extending Citizen Science. In Proceedings of the 51st Hawaii International Conference on System Sciences, 2018.

Vickie Curtis. Online citizen science games: opportunities for the biological sciences. Applied $\&$ translational genomics, 3(4):90-94, 2014.

Vickie Curtis, Richard Holliman, Ann Jones, and Eileen Scanlon. Online citizen science: participation, motivation, and opportunities for informal learning. Routledge, 2017.

Wynne Harlen. The teaching of science in primary schools. David Fulton Publishers, 2017.

KD Hassman, Gabriel Mugar, Carsten Østerlund, and Corey Jackson. Learning at the seafloor, looking at the sky: The relationship between individual tasks and collaborative engagement in two citizen science projects. In proceedings for 10th International Conference on Computer Supported Collaborative Learning, 2013.

Bruce V Lewenstein. What does citizen science accomplish. In Paper presented at the CNRS colloquium, Paris, France, 2004a.

Bruce V Lewenstein. What does citizen science accomplish. In Paper presented at the CNRS colloquium, Paris, France, 2004b.

Oded Nov, Ofer Arazy, and David Anderson. Dusting for science: motivation and participation of digital citizen science volunteers. In Proceedings of the 2011 iConference, Seattle, WA. Association for Computing Machinery, 2011.

M Jordan Raddick, Georgia Bracey, Karen Carney, Geza Gyuk, Kirk Borne, John Wallin, and Suzanne Jacoby. Citizen science: status and research directions for the coming decade. 2009.

M Jordan Raddick, Georgia Bracey, Pamela L Gay, Chris J Lintott, Carie Cardamone, Phil Murray, Kevin Schawinski, Alexander S Szalay, and Jan Vandenberg. Galaxy Zoo: Motivations of citizen scientists. arXiv preprint arXiv:1303.6886, 2013.

Jane Webster and Richard T Watson. Analyzing the past to prepare for the future: Writing a literature review. MIS Quarterly, 26(2), 2002.

Andrea Wiggins and Kevin Crowston. From conservation to crowdsourcing: a typology of citizen science. In Proceedings of the 44th Hawaii International Conference on System Sciences, 2011.

Robert Willison and Mikko Siponen. A critical assessment of IS security research between 1990-2004. In Proceedings of 15th European Conference on Information Systems, St. Gallen, Switzerland, 2007.

Poonam Yadav and John Darlington. Conceptual Frameworks for Building Online Citizen Science Projects. arXiv preprint arXiv:1704.05084, 2017. 